\title{
3 Research Square \\ Complexity In The Context of Palliative Care: A Scoping Review
}

Hironori Ohinata ( $\nabla$ hinata@iuhw.ac.jp)

Tohoku University Graduate School of Medicine

Maho Aoyama

Tohoku University Graduate School of Medicine

Mitsunori Miyashita

Tohoku University Graduate School of Medicine

\section{Research Article}

Keywords: complex, complexity, palliative care, palliative medicine, scoping review

Posted Date: October 19th, 2021

DOl: https://doi.org/10.21203/rs.3.rs-969011/v1

License: (c) (i) This work is licensed under a Creative Commons Attribution 4.0 International License.

Read Full License 


\section{Abstract}

Background: Understanding the factors of complexity of patients in palliative care is very important for healthcare providers in addressing the care needs of their patients. However, the healthcare providers' perception of the factors of complexity in palliative care lacks a common understanding. This study aimed to determine the scope of research activities and specific factors of complexity in the context of palliative care.

Methods: A scoping literature review was performed, following the methods described by the Joanna Briggs Institute. We conducted an electronic literature search in MEDLINE (Ovid), PsycINFO, Web of Science Core Collection, and CINAHL, examining literature from May 1972 to 2020.

Results: We identified 32 peer-reviewed articles published in English before 2020. The target literature mainly originated in Europe and the United States. The research methods included quantitative studies $(n=13)$, qualitative studies $(n=12)$, case studies $(n=3)$, and reviews $(n=4)$. We reviewed 32 studies and summarized the factors of complexity into three levels: the patient's level, the healthcare setting level, and the socio-cultural landscape level. We identified factors affecting patient-specific complexity, including sex, race, age, living situation, family burden, resources, treatment, decision-making, communication, prognosis, disease, and comorbidity/complexity. Other factors identified as contributing to patient complexity were the interaction of physical, psychological, social, and spiritual categories, as well as the healthcare providers' confidence and skills, and the socio-cultural components.

Conclusions: This scoping review shows specific factors of complexity and future challenges in the context of palliative care. Future research should include the factors of complexity identified in this review and conduct longitudinal studies on the interactions among them. In addition, it is necessary to examine specific complexity factors in patients from various social and ethnic backgrounds.

\section{Background}

Palliative care is a wicked problem with many incomplete, changing requirements and complex interdependencies(1). Wicked problems must be identified and strategized according to the VUCA categories of variability, uncertainty, complexity, and ambiguity(2). Some institutions use the term "complexity" in their definition of palliative care $(3,4)$. The World Health Organization defines palliative care as, "care provided to optimize the quality of life and reduce serious and complex symptoms of patients with advanced illnesses" $(5,6)$. The European Association for Palliative Care states that complexity in palliative care involves multidimensional issues caused by symptoms and problems which are difficult to manage(3). The National Coalition for Hospice and Palliative Care states that complexity is diverse, interactive, and shaped by many factors(7). Thus, the complexity of the patient's circumstances is a critical aspect of palliative care. Patients with complex needs may receive the most benefits from palliative care, especially early palliative care, including improved quality of care, patient and family satisfaction, professional satisfaction, and improved survival(8). 
However, there is a lack of common understanding of the perception of complexity(9-11). Therefore, the need to comprehend the complex circumstances of the patients who are the target of palliative care is increasing, and research on this is ongoing worldwide. Parks et al. used Bronfenbrenner's ecological systems theory to show the interaction of the factors of complexity in a framework(10). Emma et al. showed that complexity in palliative care is at the patient level, the healthcare environment, and the sociocultural landscape level(11). However, these studies show different factors of complexity. If we could recognize the factors of patient complexity in a wider and more specific context, it would contribute to timely, appropriate, individualized, and coordinated care(12). Moreover, identifying the specific factors contributing to complexity in the context of palliative care is important for identifying patients in need of these services(13). Understanding complexity is necessary for estimating palliative care needs, specialized palliative care referral and resource allocation, future intervention strategies, and consideration of non-cancer palliative care $(14,15)$. Therefore, this study aimed to determine the scope of research activities and specific factors of complexity in the context of palliative care.

\section{Methods}

\section{Literature review question}

Our research question was:

What is the scope of research activities and specific factors of complexity in the context of palliative care?

\section{Design}

We used the Joanna Briggs Institute Scoping Review framework to conduct the present scoping review(16). Furthermore, we used the PRISMA checklist to ensure the quality of the review(17).

\section{Search strategy}

We conducted first an electronic literature search in MEDLINE (Ovid), PsycINFO, Web of Science Core Collection, and CINAHL, examining literature from May 1972 to September 2020. The databases used were selected as the appropriate platforms for this review(18). The keywords and subject heading terms were "palliative care" and "complexity." The inclusion and exclusion criteria are presented in Table 1. The literature searches were initially conducted on September 24, 2020 and updated on July 7, 2021. The full MEDLINE search is available in the Supplemental Online File 1, and the other searches are available on request from the corresponding author.

Titles and abstracts were initially screened by one of the authors to identify potentially eligible papers, and any areas of uncertainty were resolved by another author. The full manuscripts of potentially eligible papers were screened by two of the authors. The two authors reached a consensus after discussion on whether there was uncertainty regarding any article's eligibility. Moreover, the third author was consulted if consensus was not reached, and a definitive list of eligible studies was agreed on (Figure 1). No 
additional hand-searching was conducted, but references of the included papers were also screened for any other relevant papers.

\section{Data extraction}

We reviewed the data to be extracted according to the Joanna Briggs Institute Reviewer's Manual(19). The researcher prepared the extraction form and one of our members checked it. We descriptively extracted data based on the country, study design, methods, type of institution, population, aim of the study, and factors of complexity of palliative care. These data were integrated and mapped using tables. The factors of complexity were classified using patient level, healthcare setting level, and socio-cultural landscape level(11). This is similar to the classification of complexity in other studies(12).

\section{Charting the data}

We constructed a research chart using the PRISMA guidelines(17). Charting the data involved developing a form to capture the characteristics of each article. Two authors extracted and summarized the data for title, author/publication date, country, study design, methods, type of institution, population (sample size), aim of the study, and factors of complexity in the context of palliative care.

\section{Data items}

We classified the factors of complexity into three levels: the patient's level, the healthcare setting level, and the socio-cultural landscape level. The patient level indicated the patients' inherent factors and the following holistic factors: physical, psychological, social, and spiritual. The healthcare setting level indicated factors related to professional skills and confidence. The socio-cultural landscape level referred to the factors related to the social and political environment.

\section{Results}

From 1,615 initial records, we screened 73 papers according to eligibility, of which 32 were included in the full review (Figure 1). A summary of the characteristics of these 32 studies is presented in Table 2.

\section{Countries and research methods}

The year of publication, country, study methods, type of institution, and population of the 32 studies included in our review are summarized in Table 3. The majority of the studies were conducted in Europe $(n=19)(8,10,11,20-34)$, followed by 7 from the USA(35-41). Studies conducted in Asia were not included. They were, however, included in the searching results, but did not meet the eligibility criteria. Two studies were published in the 1990 s(20,42), and three studies in the 2000 s(21, 35, 36). Twentyseven studies were published after 2010(8, 10,11, 22-34, 37-41, 43-47).

Quantitative methods were used in 13 studies(24, 25, 30-35, 38, 39, 41, 46, 47), while 12 were qualitative $(10,11,22,23,27-29,42-45)$. All the quantitative studies were descriptive or observational (cross-sectional, retrospective, or cohort studies). Thirteen of the studies were conducted in medical 
settings such as palliative care units or hospices $(10,11,23,24,27,31,32,35,39,41,42,45,47)$, ten were conducted by academic societies or professional teams $(22,25,28,30,33,34,38,43,46)$, and two were conducted over the telephone or at health centers for people living in specific areas $(29,44)$. Other publications were reviews or case studies that did not specify the facility where they were conducted. Eighteen studies were conducted on patients; eleven on adult or pediatric cancer patients $(21,24,25,28$, $31,32,36,38,39,42,46)$ and seven on cancer patients, as well as non-cancer patients suffering from dementia and neurological diseases $(29,30,33-35,41,47)$.

\section{Factors of complexity in palliative care}

The factors of complexity in palliative care are shown in Table 4.

Patient level of complexity. All 32 studies reported the patient level of complexity. The categories of patient-level complexity were background, physical, psychological, social, and spiritual. In addition, specific factors were described for each category. The factors of background complexity were sex $(30,33)$, race $(38,41)$, language $(10)$, age $(8,10,25,31,38,41,44,45)$, living situation $(10,11,29,44)$, family burden $(8,10,24,29,44-46)$, resources $(10,20,22,23,27,32,37,42,43)$, treatment $(11,21,33,35,39,40)$, decision-making(10, 22, 27, 33, 36, 37), communication(10, 11, 27, 32), prognosis(8, 11, 23, 25-27, 45), disease $(10,11,29,30,32-34,39,41,44,45)$, and comorbidities/multimorbidity $(8,11,24,25,27,31,38$, $39,41,44,45,47)$. Patient-level complexity was a factor for both men and women of all ages $(8,10,25$, $30,31,33,38,41,44,45)$. In English-speaking countries, non-English language barriers were a factor of complexity(10). Family burden was another factor, involving family stress and anxiety $(8,10,24,29,44-$ 46). Treatment was a factor of complexity including previous surgery, polypharmacy, and side effects of drugs $(11,21,33,35,39,40)$. Disease included progressive diseases such as heart failure, dementia, psychological disorders such as schizophrenia, and drug addiction $(10,11,29,30,32-34,39,41,44,45)$. Moreover, the presence of these diseases as comorbidities or multimorbidity was also a factor of complexity $(8,11,24,25,27,31,38,39,41,44,45,47)$. However, the studies reported multiple types and numbers of comorbidities and multimorbidity, ranging from two to five.

In the physical category of patient-level complexity, the factors were physical function $(8,24,27,31-33$, 40, 43, 47), pain $(10,11,24,25,28,29,31,33,36,43,46)$, pain treatment(21), symptoms $(11,24,27,33$, $43)$, and symptom burden $(8,31,32,36,37,40)$. Physical functioning included factors such as low performance status and disability $(24,33)$. Pain included factors such as Numeric Rating Scale $>5$ pain, refractory pain, and uncontrolled pain $(11,25)$. Pain treatment included factors such as cytokine activity and ineffective analgesics(21). Symptoms included various factors such as nausea, dyspnea, tiredness, and appetite $(11,24,27,33,43)$.

In the psychological category of patient-level complexity, the factors were anxiety $(25,30,33)$, depression(25, 33), and mental health $(8,23-25,30,40,41)$. Anxiety and depression included factors such as higher anxiety and feeling depressed $(25,30)$. Mental health included factors such as emotional distress and psychological burden $(8,24,40)$. 
In the social category, the factors were social risks $(10,11,25,29,31,32,34,36,40)$ and financial problems $(10,40)$. Social risks included factors such as social roles and family roles(32, 36). Financial problems included factors such as financial difficulties(40).

In the spiritual category of patient-level complexity, the factors were existential problems $(25,27,31)$ and spirituality $(22,40)$. Existential problems included factors such as ethical dilemmas and conflicts(25). Spirituality included factors such as spiritual needs(40).

Healthcare setting level of complexity. Fifteen studies reported the healthcare setting level of complexity $(8,10,11,20,22,23,25-27,29,32,34,41,44)$. In relation to the role of healthcare providers, the factors of complexity included truth-telling(20), advocacy(20), and differing or conflicting priorities of care and treatment(27). In addition, the role of the nurse included demands for emotional support, skills, and confidence $(11,20,26)$.

Socio-cultural landscape level of complexity. Four studies reported the socio-cultural landscape level of complexity $(10,11,40)$. Social capital, which includes the systems that support the interrelationships and connections among people in a society or local community, was a factor of complexity(11). Other factors of complexity included local and national cultures, as well as societal cultures which might affect the patients(40). The cultural factor was the value of the end of life in society; this was a factor in the structuring of health and social systems of care(10).

Interaction of the factors of complexity in palliative care. Ten studies reported interactions of the factors $(8-11,21,31-33,40,42)$. In all studies, patient complexity arose from the interaction of physical, psychological, social, and spiritual factors. Hodiamont et al. showed the interaction and dynamics of the factors of complexity(9). Interactions of factors of complexity were reported not only at the patient level, but also at the healthcare setting level and the socio-cultural landscape level(10,11). Park et al. reported that interactions among healthcare providers and care settings can make patients better or worse(10). Tuca et al. also reported that the interventions that healthcare providers provide to patients result in outcomes that are more than the sum of the parts(25).

\section{Discussion}

This scoping review reported on the scope of research activities and specific factors of complexity in the context of palliative care. The main findings of the review are as follows: 1 ) we identified specific factors of complexity at the patient level, the healthcare setting level, and the socio-cultural landscape level; 2) there is a need to investigate the interrelationships of the factors of complexity.

First, this review included studies that were conducted using various methods: quantitative studies, qualitative studies, case studies, and reviews. Our improved understanding of the factors of complexity in palliative care is necessary in order to address the specific complex needs of patients $(11,40)$. In our review, the patients' inherent factors were those related to sex, race, age, living situation, family burden, resources, treatment, decision-making, communication, prognosis, disease, and 
comorbidity/multimorbidity. However, not all of these factors are considered complex for patients in palliative care(10,33); what is complex depends on each patient's situation. For example, different diseases have different factors of complexity(34). In cancer patients, several physical symptoms deteriorate simultaneously at the end of life(48). Therefore, the factor of complexity increases at the end of life and more complex needs of the patient arise. Whereas, patients with dementia show a gradual decline in physical and mental functions(49). A decline in cognitive function makes communication difficult. The trajectory of such chronic diseases has been reported to be different in cancer and noncancer patients(50). Furthermore, the treatment approach for pain in patients with cancer is different from that in patients with other diseases(51). In our review, many studies were conducted with cancer patients. Therefore, factors of complexity in palliative care identified here are potentially biased toward patients with cancer, and further research is needed to identify factors of complexity specific to patients with other diseases.

Also, assessment instruments would help in identifying patients' complex needs. Assessment instruments include Hexagon of Complexity (HexCom) and Instrument Diagnosing Complexity in Palliative Care (IDC-Pal) $(8,34)$. HexCom assesses the complexity of a patient's condition in six domains (clinical, spiritual, social/family, spiritual, ethical, and death-related), as well as in terms of resources. Researchers can share a common understanding of the factors of complexity of patients using these assessment instruments(8). However, in this review, the social and spiritual factors of complexity were not described in any specific situation. The social and spiritual factors are formed in the cultural context of national and local cultural values and ethnic history(52). Therefore, future studies are needed to identify the factors of complexity in palliative care in various regions and their specific cultures.

Second, there is a need to investigate the interactions of complexity in palliative care. Our review identified that at the patient level; physical, psychological, social, and spiritual factors as well as background factors interact. In addition, factors of complexity interact at the healthcare setting level and at the socio-cultural landscapes level(11). Safford et al. showed the interaction between biological, socioeconomic, cultural, environmental, and behavioral forces in a vector model of complexity(53). The vector model can harmonize the delivery of healthcare with complexity, given the influences among the factors. However, the complex needs in palliative care are not structured in how they change because of the interaction between factors(54). Nevertheless, "Phase of Illness" may be useful to capture the changing complexity of patients. Phase of illness can be divided into stable, unstable, deteriorating, terminal, and bereavement phases, and is a useful tool for assessing the complexity of patient needs and the resources needed to meet them(55). For example, when a patient changes from stable to unstable, the Integrated Palliative Care Outcome Scale and the physical functioning scale could be assessed to determine the patient's detailed needs $(56,57)$. Thus, the Phase of Illnesses may be helpful in ensuring that patients receive the right care at the right time, in the right place(58). Therefore, longitudinal studies that follow changes in Phases of Illnesses and patients' complexity levels over time are needed to understand the interaction of factors of complexity. Furthermore, complexity in palliative care is not only a patient factor, but also a healthcare provider factor(32). The skills of the healthcare provider, for example, in selecting analgesics and treatment algorithms for refractory pain, can address the physical 
factor of complexity(59). Thus, patients may benefit greatly if we could ascertain how to cope with some conditions from the interaction of factors that change without order.

\section{Strengths and limitations}

This scoping review followed established systematic methods and investigated factors of complexity in the context of palliative care. It reviewed included studies from different countries and socio-cultural regions. However, this review had certain limitations. There was no manual search of major journals or gray literature. The definition of palliative care was qualitatively defined rather than using specific staging criteria, and we did not perform additional searches using "complexity" as the primary search term. In addition, we only reviewed articles that were published in English. Given all these factors, some data sources may have been overlooked. We did not focus on the patterns of interaction of the various factors of complexity, but we acknowledge that these are important areas and will be the focus of future research. Finally, within the scope of this review, we did not include the quality assessment of all the studies included, since the risk of bias or evaluation is not recommended.

\section{Conclusion}

Our scoping review shows the scope of research activities and specific factors of complexity in the context of palliative care. Thirty-two studies were included in this review. We identified specific factors of complexity at the patient level, at the healthcare setting level, and at the socio-cultural landscapes level. We suggest future studies to investigate the factors that contribute to complexity in palliative care for patients with non-cancer diseases. We also need to investigate how the factors of complexity interact with each other. We share a common understanding of complexity, which could help us make early and appropriate interventions and reduce the complexity of the patient.

\section{Abbreviations}

VUCA: variability, uncertainty, complexity, and ambiguity HexCom: Hexagon of Complexity IDC-Pal: Instrument Diagnosing Complexity in Palliative Care

\section{Declarations}

\section{Authors' contributions}

$\mathrm{HO}$ made a substantial contribution to the design of this study and drafted the article. MA interpreted the data and revised it critically for important intellectual content. MM contributed to the concept or design of this study and revised it critically for important intellectual content. All authors read and approved the final manuscript.

\section{Competing interests}


The authors declare that they have no competing interests.

\section{Funding}

This work was supported by MHLW Cancer Research Program Grant Number 19EA1011, and JSPS KAKENHI (Grant No. JP 18K17480)

\section{Availability of data and materials}

All data generated or analyzed during this study are included in this published article and its supplementary information files.

\section{Ethics approval and consent to participate}

As this study represents a scoping literature review, formal ethics approval was not required.

\section{Consent for publication}

Not applicable.

\section{Acknowledgements}

Not applicable.

\section{References}

1. Periyakoil VS. Taming wicked problems in modern health care systems. J Palliat Med. 2007;10(3):658-9.

2. Bennett N, Lemoine J. What VUCA really means for you. Harv Bus Rev. 2014;92(1/2).

3. Radbruch L, Payne S. White paper on standards and norms for hospice and palliative care in Europe: part 1. Eur J Palliat Care. 2009;16(6):278-89.

4. Care. NCPfQP. Clinical Practice Guidelines for Quality Palliative Care, 4th edition. Richmond, VA: National Coalition for Hospice and Palliative Care. 2018; https://www.nationalcoalitionhpc.org/ncp.

5. World Health Organiation. Integrating palliative care and symptom relief into primary health care: a WHO guide for planners, implementers and managers. Geneva: World Health Organization; 2018.

6. Quill TE, Periyakoil VS, Denney-Koelsch EM, et al. Primer of palliative care, 7th Edition. AAHPM; 2019.

7. Ferrell BR, Twaddle ML, Melnick A, Meier DE. National Consensus Project Clinical Practice Guidelines for Quality Palliative Care Guidelines, 4th Edition. J Palliat Med. 2018;21(12):1684-89.

8. Martin-Rosello ML, Sanz-Amores MR, Salvador-Comino MR. Instruments to evaluate complexity in end-of-life care. Curr Opin Support Palliat Care. 2018;12(4):480-88.

9. Hodiamont F, Junger S, Leidl R, et al. Understanding complexity - the palliative care situation as a complex adaptive system. BMC Health Serv Res. 2019;19(1):157. 
10. Pask S, Pinto C, Bristowe K, et al. A framework for complexity in palliative care: A qualitative study with patients, family carers and professionals. Palliat Med. 2018;32(6):1078-90.

11. Carduff E, Johnston S, Winstanley C, et al. What does 'complex' mean in palliative care? Triangulating qualitative findings from 3 settings. BMC Palliat Care. 2018;17(1):12.

12. Schaink AK, Kuluski K, Lyons RF, et al. A scoping review and thematic classification of patient complexity: offering a unifying framework. J Comorb. 2012;2:1-9.

13. Weissman DE, Meier DE. Identifying patients in need of a palliative care assessment in the hospital setting: a consensus report from the Center to Advance Palliative Care. J Palliat Med. 2011;14(1):1723.

14. Hui D, Mori M, Watanabe SM, et al. Referral criteria for outpatient specialty palliative cancer care: an international consensus. Lancet Oncol. 2016;17(12):e552-e559.

15. Gemmell R, Yousaf N, Droney J. "Triggers" for early palliative care referral in patients with cancer: a review of urgent unplanned admissions and outcomes. Support Care Cancer. 2020;28(7):3441-49.

16. Peters MDJ, Godfrey CM, Khalil H, et al. Guidance for conducting systematic scoping reviews. Int J Evid Based Healthc. 2015;13(3):141-46.

17. Tricco AC, Lillie E, Zarin W, et al. PRISMA extension for scoping reviews (PRISMA-ScR): checklist and explanation. Ann Intern Med. 2018;169(7):467-73.

18. Bramer WM, Rethlefsen $\mathrm{ML}$, Kleijnen J, Franco OH. Optimal database combinations for literature searches in systematic reviews: a prospective exploratory study. Syst Rev. 2017;6(1):245.

19. Peters MD, Godfrey C, Mclnerney P, et al. Scoping reviews. Joanna Briggs Institute Reviewer's Manual. 2017:408-46.

20. Morrissey MV. Extending the theory of awareness contexts by examining the ethical issues faced by nurses in terminal care. Nurs Ethics. 1997;4(5):370-9.

21. Hemming L, Maher D. Cancer pain in palliative care: why is management so difficult? Br J Community Nurs. 2005;10(8):362-7.

22. Pastrana T, Radbruch L, Nauck F, et al. Outcome indicators in palliative care-how to assess quality and success. Focus group and nominal group technique in Germany. Support Care Cancer. 2010;18(7):859-68.

23. Quinn C, Bailey ME. Caring for children and families in the community: experiences of Irish palliative care clinical nurse specialists. Int J Palliat Nurs. 2011;17(11):561-7.

24. Gaertner J, Frechen S, Sladek M, Ostgathe C, Voltz R. Palliative care consultation service and palliative care unit: why do we need both? Oncologist. 2012;17(3):428-35.

25. Tuca-Rodriguez A, Gomez-Batiste X, Espinosa-Rojas J, et al. Structure, organisation and clinical outcomes in cancer patients of hospital support teams in Spain. BMJ Support Palliat Care. 2012;2(4):356-62.

26. Watts T. Initiating end-of-life care pathways: a discussion paper. J Adv Nurs. 2012;68(10):2359-70. 
27. Axelsson L, Klang B, Lundh Hagelin C, Jacobson SH, Gleissman SA. End of life of patients treated with haemodialysis as narrated by their close relatives. Scand J Caring Sci. 2015;29(4):776-84.

28. Hackett J, Godfrey M, Bennett MI. Patient and caregiver perspectives on managing pain in advanced cancer: A qualitative longitudinal study. Palliat Med. 2016;30(8):711-19.

29. de Veer AJE, Stringer B, van Meijel B, Verkaik R, Francke AL. Access to palliative care for homeless people: complex lives, complex care. BMC Palliat Care. 2018;17(1):119.

30. Mateo-Ortega D, Gomez-Batiste X, Mate J, et al. Effectiveness of psychosocial interventions in complex palliative care patients: A quasi-experimental, prospective, multicenter study. J Palliat Med. 2018;21(6):802-8.

31. Tuca A, Gomez-Martinez M, Prat A. Predictive model of complexity in early palliative care: a cohort of advanced cancer patients (PALCOM study). Support Care Cancer. 2018;26(1):241-49.

32. Carrasco-Zafra MI, Gomez-Garcia R, Ocana-Riola R, Martin-Rosello ML, Blanco-Reina E. Level of palliative care complexity in advanced cancer patients: A multinomial logistic analysis. J Clin Med. 2020;9(6):1960.

33. Kamal AH, Check DK, Bull J, et al. Associations of patient characteristics and care setting with complexity of specialty palliative care visits. J Palliat Med. 2020;24(1).

34. Busquet-Duran X, Jiménez-Zafra EM, Manresa-Domínguez JM, et al. Describing complepity in palliative home care through hexcom: A cross-sectional, multicenter study. J Multidiscip Healthc. 2020;13:297-308.

35. Cowan JD, Walsh D, Homsi J. Palliative medicine in a United States cancer center: a prospective study. Am J Hosp Palliat Care. 2002;19(4):240-50.

36. Hinds PS, Oakes LL, Hicks J, Anghelescu DL. End-of-life care for children and adolescents. Semin Oncol Nurs. 2005;21(1):53-62.

37. Reid TT, Demme RA, Quill TE. When there are no good choices: illuminating the borderland between proportionate palliative sedation and palliative sedation to unconsciousness. Pain Manag. 2011;1(1):31-40.

38. Phongtankuel V, Johnson P, Reid MC, et al. Risk factors for hospitalization of home hospice enrollees development and validation of a predictive tool. Am J Hosp Palliat Care. 2017;34(9):806-13.

39. Thrane SE, Maurer SH, Cohen SM, May C, Sereika SM. Pediatric palliative care: A five-year retrospective chart review study. J Palliat Med. 2017;20(10):1104-11.

40. Burt M, Kamal AH. Practical strategies for optimizing and integrating palliative care in cancer. Curr Oncol Rep. 2018;20(12):97.

41. Battisti KA, Cohen DM, Bourgeois T, Kline D, Zhao S, lyer MS. A paucity of code status documentation despite increasing complex chronic disease in pediatrics. J Palliat Med. 2020;23(11):1452-59.

42. Bottorff JL, Steele R, Davies B, et al. Striving for balance: palliative care patients' experiences of making everyday choices. J Palliat Care. 1998;14(1):7-17. 
43. Johnson C, Paul C, Girgis A, Adams J, Currow DC. Australian general practitioners' and oncology specialists' perceptions of barriers and facilitators of access to specialist palliative care services. $J$ Palliat Med. 2011;14(4):429-35.

44. McVey P, McKenzie H, White K. A community-of-care: the integration of a palliative approach within residential aged care facilities in Australia. Health Soc Care Community. 2014;22(2):197-209.

45. Oliveira I, Fothergill-Bourbonnais F, McPherson C, Vanderspank-Wright B. Battling a tangled web: The lived experience of nurses providing end-of-life care on an acute medical unit. Res Theory Nurs Pract. 2016;30(4):353-78.

46. Pidgeon T, Johnson CE, Currow D, et al. A survey of patients' experience of pain and other symptoms while receiving care from palliative care services. BMJ Support Palliat Care. 2016;6(3):315-22.

47. Santos CED, Klug D, Campos $L$, et al. Analysis of the Perroca scale in palliative care unit. Rev Esc Enferm USP. 2018;52:e03305.

48. Teunissen SC, Wesker W, Kruitwagen C, et al. Symptom prevalence in patients with incurable cancer: a systematic review. J Pain Symptom Manage. 2007;34(1):94-104.

49. Murray SA, Kendall M, Mitchell G, et al. Palliative care from diagnosis to death. BMJ. 2017;356:j878.

50. Lynn J, Adamson DM. Living Well at the End of Life: Adapting Health Care to Serious Chronic Illness in Old Age. RAND Corporation; 2003.

51. Turk DC, Wilson HD, Cahana A. Treatment of chronic non-cancer pain. Lancet (London, England). 2011;377(9784):2226-2235.

52. Leininger M, Reynolds C. Culture care diversity and universality theory. Nursing. 1991.

53. Safford MM, Allison JJ, Kiefe Cl. Patient complexity: more than comorbidity. the vector model of complexity. J Gen Intern Med. 2007;22(Suppl 3):382-90.

54. Gray B. The Cynefin framework: applying an understanding of complexity to medicine. Journal of Primary Health Care. 2017;9(4):258-61.

55. Mather H, Guo P, Firth A, et al. Phase of Illness in palliative care: Cross-sectional analysis of clinical data from community, hospital and hospice patients. Palliat Med. 2018;32(2):404-12.

56. Eagar K, Watters P, Currow DC, Aoun SM, Yates P. The Australian Palliative Care Outcomes Collaboration (PCOC)-measuring the quality and outcomes of palliative care on a routine basis. Australian Health Review. 2010;34(2):186-92.

57. Guo P, Dzingina M, Firth AM, et al. Development and validation of a casemix classification to predict costs of specialist palliative care provision across inpatient hospice, hospital and community settings in the UK: a study protocol. BMJ Open. 2018;8(3):e020071.

58. Murtagh F. Complexity, case-mix and outcomes - emerging UK evidence. 2014; https://www.kcl.ac.uk/cicelysaunders/attachments/fliss-murtagh-presentation.pdf. Accessed 7, 2021.

59. Tassinari D, Drudi F, Rosati M, Maltoni M. Transdermal opioids as front line treatment of moderate to severe cancer pain: a systemic review. Palliat Med. 2011;25(5):478-87. 


\section{Tables}

Due to technical limitations, tables $1,2,3$ and 4 are only available as a download in the Supplemental Files section.

\section{Figures}

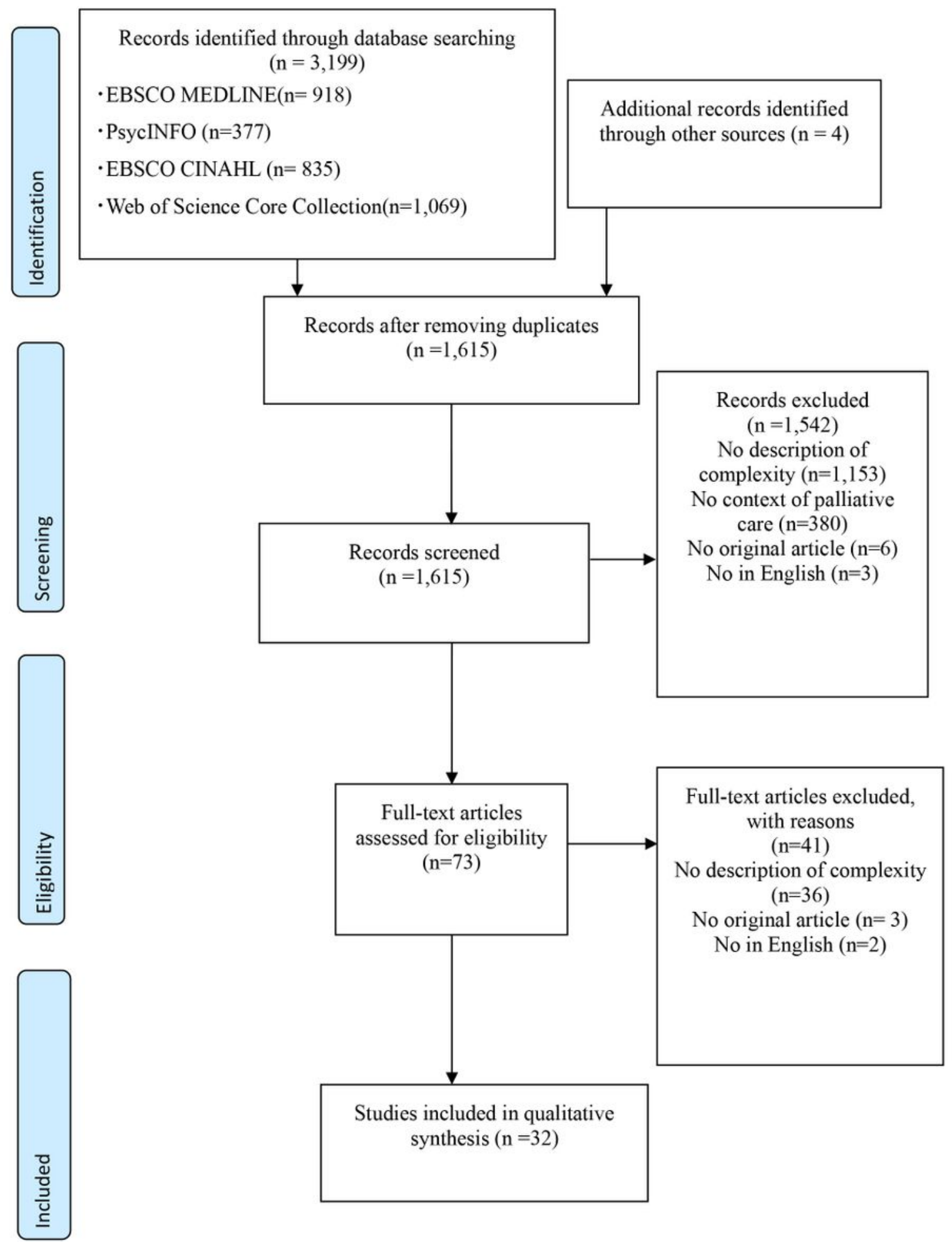




\section{Figure 1}

Flow diagram for the scoping review process.

\section{Supplementary Files}

This is a list of supplementary files associated with this preprint. Click to download.

- $\sup 1 . x \mid s x$

- complexityreview.xlsx 